\title{
Physics of Traffic and the Crowds Theory: A Multidisciplinary Approach to Mobility Education
}

\author{
Laura Tamburini $^{1,2}$ and Sabrina Colomban ${ }^{3}$ \\ 1. Regional Board of Education, Friuli Venezia Giulia 34124, Italy \\ 2. Department of Life Sciences, University of Trieste, Trieste 34100, Italy \\ 3. Traffic Police Department, Traffic Police, Trieste 34100, Italy
}

\begin{abstract}
Crowding is a reality in which we find ourselves involved daily. The crowd produced in a traffic jam is a dynamic entity in which the application of physics, mathematics and biology can provide practical help to understand how and why this problematic situation occurs and what solutions can be found to resolve it. In the mobility education project "SicuraMENTE", we carried out an experiment on the conduct of a crowd by simulating a situation of intense city traffic. Taking a cue from an experimental situation proposed in traffic physics, we have verified that a route with limited access can generate a traffic jam and the crowd of pedestrians can be mitigated by forcing the crowd to use dedicated streets. Taking the outgoing time of the student crowd from the outlet road, with and without the presence of an obstacle in the middle of the roadway, it was found that the traffic jam is resolved more quickly in the situation with an obstacle because the flow of people is divided into two separate channels, reducing the probability that two individuals are close and that this creates an obstruction (example of counter-intuitive physical principle). We also verified that the speed of the elements of the crowd influences the formation of traffic jams, which are on average more likely in the case of higher speed. These important aspects in the design of road networks and transport infrastructure have made students reason on physics' topics, but also on the correct conduct in traffic. The multidisciplinary approach in education for safe and sustainable mobility, so innovative in Italy, turned out to be effective in terms of teaching in the frame of a mix of academic disciplines, in which road safety education has become the context and the goal.
\end{abstract}

Key words: Mobility education, physics of traffic, crowd theory, middle school.

\section{Introduction}

We find ourselves involved in crowing daily: at the supermarket, waiting in the queue at a counter, in the cinema, but especially in traffic. In a traffic jam, the crowd is a dynamic entity, a fluid-mechanical system in which the application of physics, mathematics and biology can provide practical help to understand how and why problematic situations occur and what solutions can be found to resolve it. Pedestrian traffic flows can be treated similarly to vehicle traffic flows $[1,2]$, where the current modeling of crowds uses two possible approaches to the study of such a complex system: the microscopic approach, which studies the individual elements, and the macroscopic one, which

Corresponding author: Laura Tamburini, Ph.D. student, professor, research fields: neural and cognitive sciences. E-mail: laura.tamburini1@gmail.com. studies the evolution of a large-scale system [3, 4].

The first one involves individual units with characteristics such as individual speed and individual interaction [5, 6] and uses difficult and expensive mathematical simulation. Another microscopic approach, called pedestrian analysis, is being increasingly used instead of the mathematical models [7]. In such models, pedestrians are treated as discrete individuals moving in a computer simulated environment.

The macroscopic model is concerned with average behavior, such as traffic density, average speed and module area: it is computationally less expensive because this approach has fewer design details in terms of interaction among vehicles and between vehicles and their environment [3, 8-10]. Above a critical density, the crowd no longer moves as a set of 
individuals, but behaves like a fluid spread over the space. Multiple interactions occur within a crowd among single elements that may produce different behaviors that can fluctuate rapidly: the elements stop behaving independently (flock effect) or avoid contact with adjacent elements (similar to the electron-electron repulsion) [11]. The external conditions are important for the conduct of the crowd, which is brought to react to the surrounding environment. The variations of the space can alter its behavior.

Since 2010, the didactic project called "SicuraMENTE" was carried out in Italian region Friuli Venezia Giulia (in the north-eastern part of Italy) as a partnership between the Regional Board of Education-Ministry for Education, University and Research and the regional government, in collaboration with the Traffic Police of Trieste and FVG (Friuli Venezia Giulia) Roads Ltd. (managers of state highways in Friuli Venezia Giulia). In this project, where the mobility education is offered by the schools inside the curricular disciplines, some core activities concerning road safety education from the scientific and social point of view were proposed to primary and middle schools: The rationale was a perspective of road safety education as both a scientific education and a citizenship education, establishing the scientific and social meaning of rules as a basic step to make students follow them.

In 2013, we carried out an experiment on the conduct of a crowd by simulating a situation of intense city traffic. After attending some lectures given by the traffic police on the traffic rules, 45 pupils from two 2 nd classes of a middle school in Trieste (the capital of Friuli Venezia Giulia) designed and built an articulated route path in the courtyard of their school, complete with road markings and vertical signs for walking and cycling. Taking a cue from an experimental situation proposed by Katsuhiro Nishinari of the Faculty of Engineering, University of Tokyo (Japan) and by Timothy Saunders of European
Molecular Biology Laboratory in Heidelberg (Germany), we verified that a route with limited access (outlet to closed path) can generate a traffic jam and the crowd of pedestrians can be eased by forcing the crowd into dedicated streets. Taking the outgoing time of the student crowd from the outlet road, with and without the presence of an obstacle placed in the middle of the roadway, it was found that the traffic jam is resolved more quickly in the situation with an obstacle because the flow of people is divided into two separate channels, reducing the probability that two individuals are close and that this creates an obstruction (example of a counter-intuitive physical rule). Comparing the output times with and without an obstacle where the students came from the route by walking or running, we verified that the speed of the elements of the crowd influences the formation of traffic jams, which are on average more likely in the case of higher speeds.

The fact that an obstacle accelerates exiting from a bounded path, and that the increasing speed of the crowd results in a higher probability of traffic jam-important aspects in the design of road networks and transport infrastructure-made students reason about the aspects of physics of fluids, but also about the correct conduct in traffic.

\section{Interdisciplinary in Mobility Education}

In Italy, road safety education is mandatory at schools on all levels [12], but teachers do not know how and what to teach students. In primary and secondary schools, there is a lack of comprehensive mobility education (at least a few hours of traffic safety education in the frame of school lessons would be welcome). In the "SicuraMENTE" project, education for safe and sustainable mobility was introduced across different disciplines (physics, geography, citizenship education, etc.). In particular, physics is usually not taught in a disconnected way from reality, but in relation to the context [13]. Road safety education can be considered an appropriate 
context for establishing a continuous link among different subjects with a collection of curricular proposals involving different school levels and for producing innovation in teaching methods to promote active involvement of pupils in learning by laboratory activities [14]. Context and scientific learning are linked in several ways: learning is contextualized (being shaped by the phenomenological context of learning) and motivation for learning implies personal involvement of the students in this context [14-17]. It is increasingly necessary to provide a large context and, in particular, scientific knowledge should be placed not only in subject content related contexts but also in socio-cultural contexts. So, there is a need for designing, proposing and revising learning physics land to integrate it into social issues. Among these, a rich context is road safety education, where the scientific concepts play a crucial role. Physical concepts are hinted to some cases but they do not reach the value of conceptual knowledge.

Before we started the project "SicuraMENTE", good examples of activities related to context-based physics learning and concerning traffic safety were proposed [18-22]. From these projects, it becomes obvious that there is a great need to study how the conceptual knowledge of physics can be integrated in science and society contexts, thus, grounding a new way of looking at the setting of both physics and science and society, not evoked by, but based on the fact that subjective knowledge is constructed.

The study of motion and of relative motions is the prelude to the proposed analysis of the safety distance, trajectory and position, displacement and velocity vectors are basic kinematic quantities to explain to students. Human reaction time and dynamic parameters of the motion on the road, such as momentum and sliding and rolling friction on the road, have also been introduced in this context.

Physics of traffic is a field that relates to many topics connected to safe mobility, too.

The crowd that produces a traffic jam is a dynamic entity, a fluid-mechanical system in which physics and mathematics provide practical help for understanding how and why problematic situations like jams occur and what solutions can be found to solve such problems. Many analogies exist between the interaction in the crowds (flock effect, elements repulsion, etc.) and other physical concepts (fluid flow, propagation of shock waves, electron-electron repulsion, etc.).

\section{Experimental Design}

An experiment carried out in a biological context [23] and in a physical context [24, 25] was adapted and contextualized in an educational context. There, the presence of an obstacle located at a certain distance from an exit, allowed to reduce total evacuation time of a crowd present within a room [26, 27].

This phenomenon is interpreted by assuming that, in the absence of an obstacle, many interactions are generated among the crowd's elements [28], providing barrier effects.

The absence of an obstacle near the only exit causes a high number of conflicts among the elements. The presence of an obstacle in a centered position relative to the outlet instead reduces such conflicts, forcing the crowd into dedicated streets (Figs. 1 and 2). If the obstacle is shifted into a lateral position relative to the outlet, however, the conflict is not resolved (Fig. 3).

In analogy to this, we assume that a route with limited outflow can generate a traffic jam (of pedestrians) and that the crowd of pedestrians trying to use an exit can be leased up by forcing the crowd into dedicated paths.

\subsection{Material and Methods}

\subsubsection{Experimental Design}

After attending some lectures given by traffic police on the traffic rules, some pupils of a junior high school in Triest (Italy) designed and built an articulated route path in the courtyard of their school, 
Effects of an obstacle near an exit
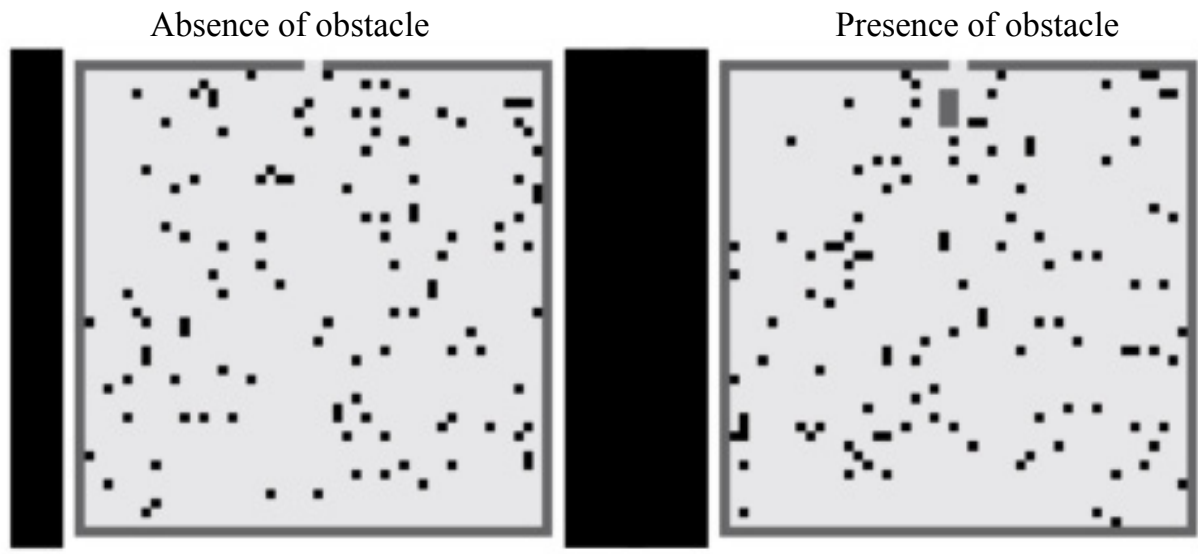

Total evacuation time: $62 \mathrm{~s}$ (absence); $55 \mathrm{~s}$ (presence)

Size of the room $25 \mathrm{~m} \times 25 \mathrm{~m}, 125$ persons

Total evacuation time is reduced by an obstacle

Fig. 1 Evacuation time with and without obstacle [24].

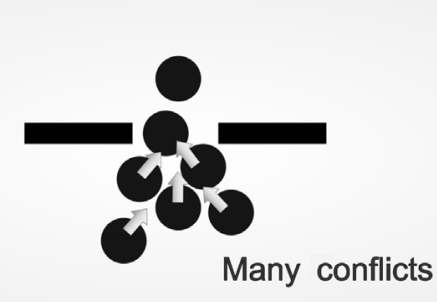

(a)

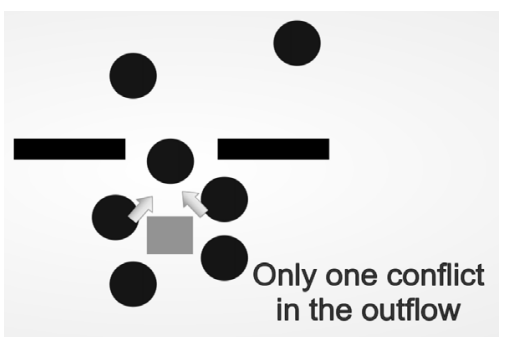

(b)

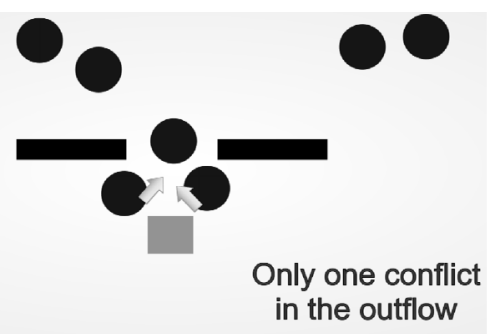

(c)

Fig. 2 Conflicts with and without a centered obstacle: (a) many conflicts without obstacle; (b) only one conflict with obstacle; (c) only one conflict with obstacle.

Source: by M. Conti.

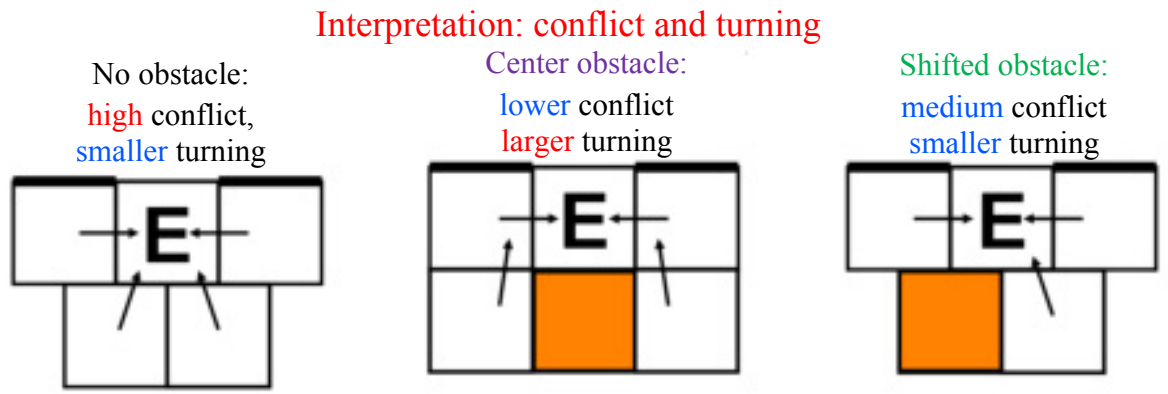

Fig. 3 Conflicts and turning without obstacle, with centered obstacle and shifted obstacle [24].

complete with road markings and vertical signs, for walking and cycling.

\subsubsection{Sample}

Forty-six students (males and females, 12-13 years old of two 2nd classes of junior high school "Campi Elisi" in Triest) took part in the experiment.
The path realized by the children consists of a rectangular space of $6.00 \mathrm{~m}$ wide by $4.00 \mathrm{~m}$ long with a $3.00 \mathrm{~m}$ width exit. The path's area is $24 \mathrm{~m}^{2}$ and the students' density is $0.5 / \mathrm{m}^{2}$. The obstacle is a foam-rubber cube. The size of the cube is $0.70 \mathrm{~m} \times$ $0.70 \mathrm{~m}$ and it is placed at a distance of $1 \mathrm{~m}$ from the 
path's exit (Fig. 4).

Before the experiment's implementation, students were given a questionnaire with 15 multiple choice items in which we asked them to make predictions on the exit times to be expected in the following cases:

- walking in the absence of obstacle $\left(t_{1}\right)$;

- walking in the presence of obstacles $\left(t_{2}\right)$;

- running in the absence of obstacle $\left(t_{3}\right)$;

- running in the presence of obstacle $\left(t_{4}\right)$.

The children's responses to the questionnaire were:

- $t_{1}>t_{2}$ and $t_{3}>t_{4}$ (95\% of cases);

- $t_{1}>t_{2}$ and $t_{3}>t_{4}$ (98\% of cases).

Then the four experiments were conducted and exit times $t_{1}$ and $t_{2}$ (in walking mode) and $t_{3}$ and $t_{4}$ (in running mode) were measured with a stopwatch (Fig. 5). Each of the four cases was repeated for three times in order to calculate the average value of the exit time (Table 1).

\subsection{Results and Discussions}

When comparing the output times, with and without obstacle, in the case where the students came from the route walking (first step) or running (second step), we verified that the speed of the elements of the crowd influences the formation of traffic jams, which are on average more likely in the case of higher speed (Fig. 6).

The fact that an obstacle accelerates the exit rate from a closed path, and that the increasing speed of the crowd results in a higher probability of a traffic jam-important aspects in the design of road networks and transport infrastructure-made students think about the aspects of the physics of fluids, but also about the correct conduct in traffic.

Contrary to predictions of the children, experimental data show that exit times are shorter with the cube, both in walking and in running mode. The presence of an obstacle makes the exit of the crowd more rapid because it reduces the interactions between the individuals.

The concept that an obstacle speeds up the exit of a crowd is a concept of counter-intuitive physical facts.

Another concept that involves the dynamic output of the crowd resulting from the experimental data is that running decreases the exit-time but increases the likelihood of element collisions and thus traffic jams.

At the conclusion of the presentation and after the discussion of the experimental data, a second questionnaire with 20 items about correct behavior in traffic is administered to the students. The most significant results related to road safety that emerged from the students' answers are:

- High initial speed can produce traffic jams $(86 \%$ of cases);

- It is not always an advantage to be fast in traffic, especially in tight situations (93\% of cases);

- High speed increases the risk of collisions $(88 \%$ of cases).

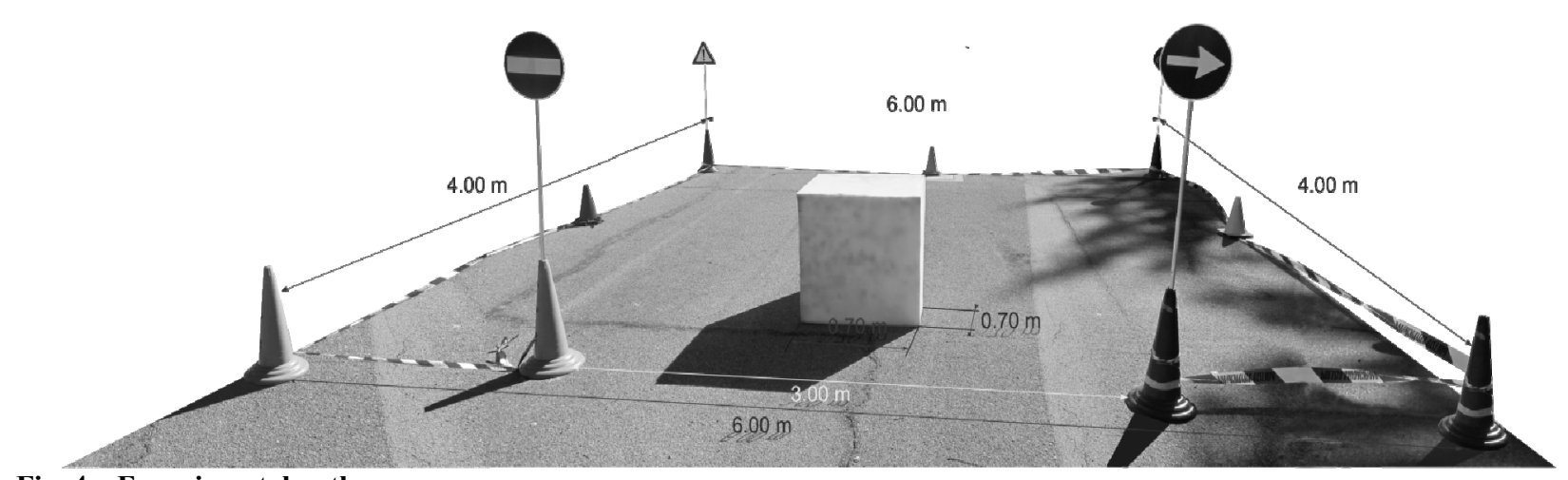

Fig. 4 Experimental path.

Source: photo by M. Conti. 

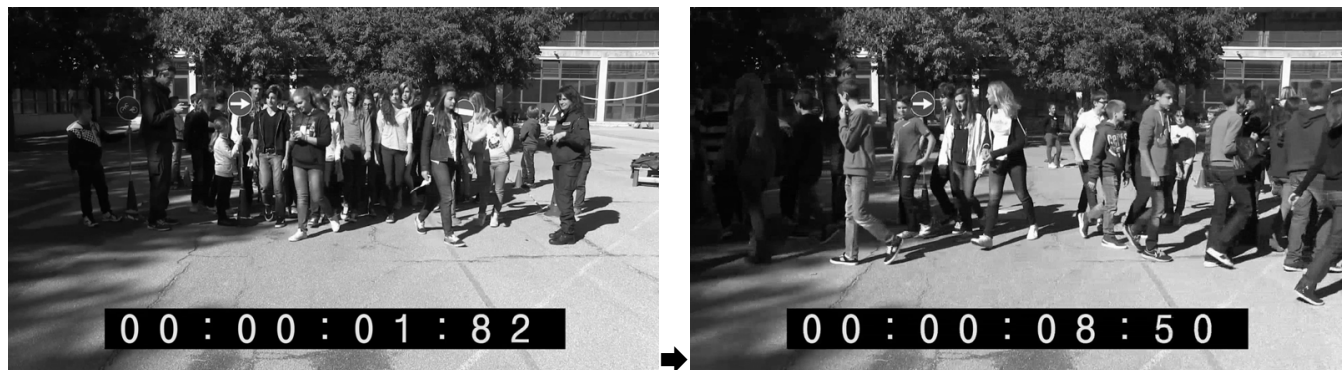

(a)
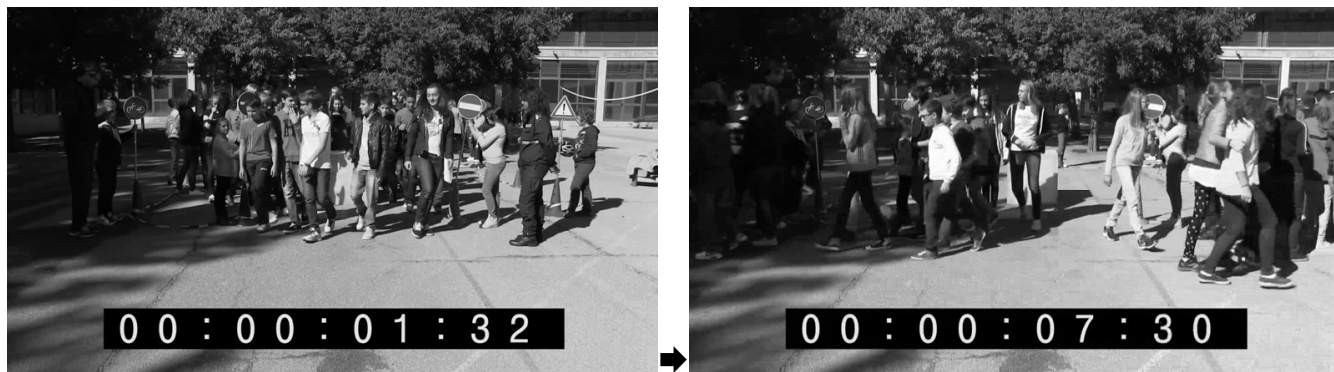

(b)
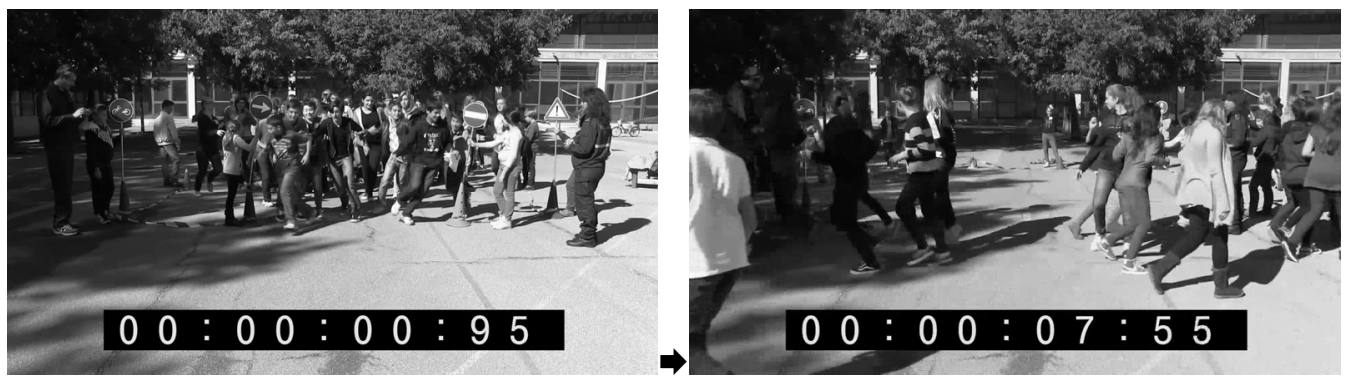

(c)
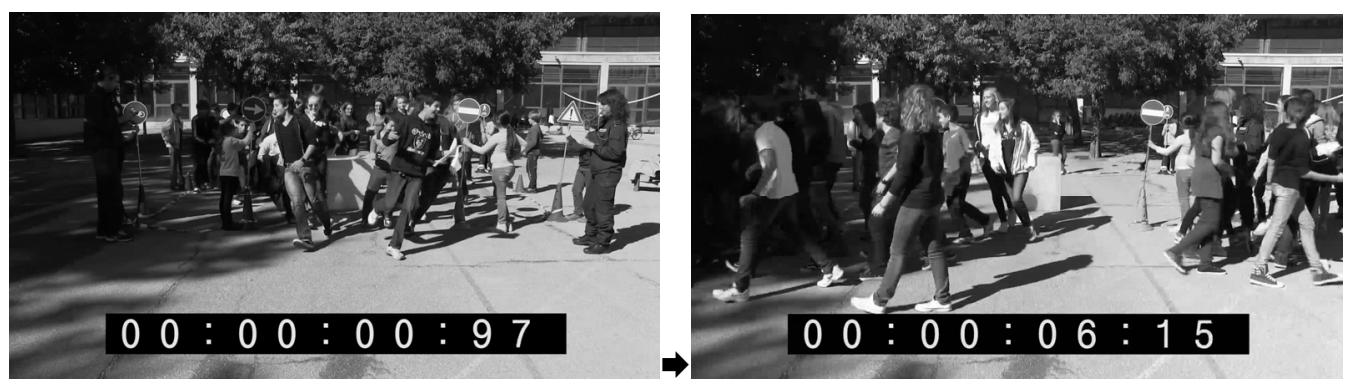

(d)

Fig. 5 The experiment with students: (a) 1st step: walking-without cube; (b) 1st step: walking—with cube; (c) 2nd step: running-without cube; (d) 2nd step: running —with cube. Source: photos by M. Conti.

Table 1 Students evacuation time.

\begin{tabular}{lllll}
\hline Total evacuation time (s) & 1st misure & 2nd misure & 3rd misure & Average \\
\hline$t_{1}$ & 8.90 & 8.50 & 8.20 & 8.60 \\
$t_{2}$ & 7.10 & 6.95 & 7.25 & 7.30 \\
$t_{3}$ & 7.50 & 7.80 & 7.35 & 7.55 \\
$t_{4}$ & 6.05 & 6.30 & 6.10 & 6.15 \\
\hline
\end{tabular}




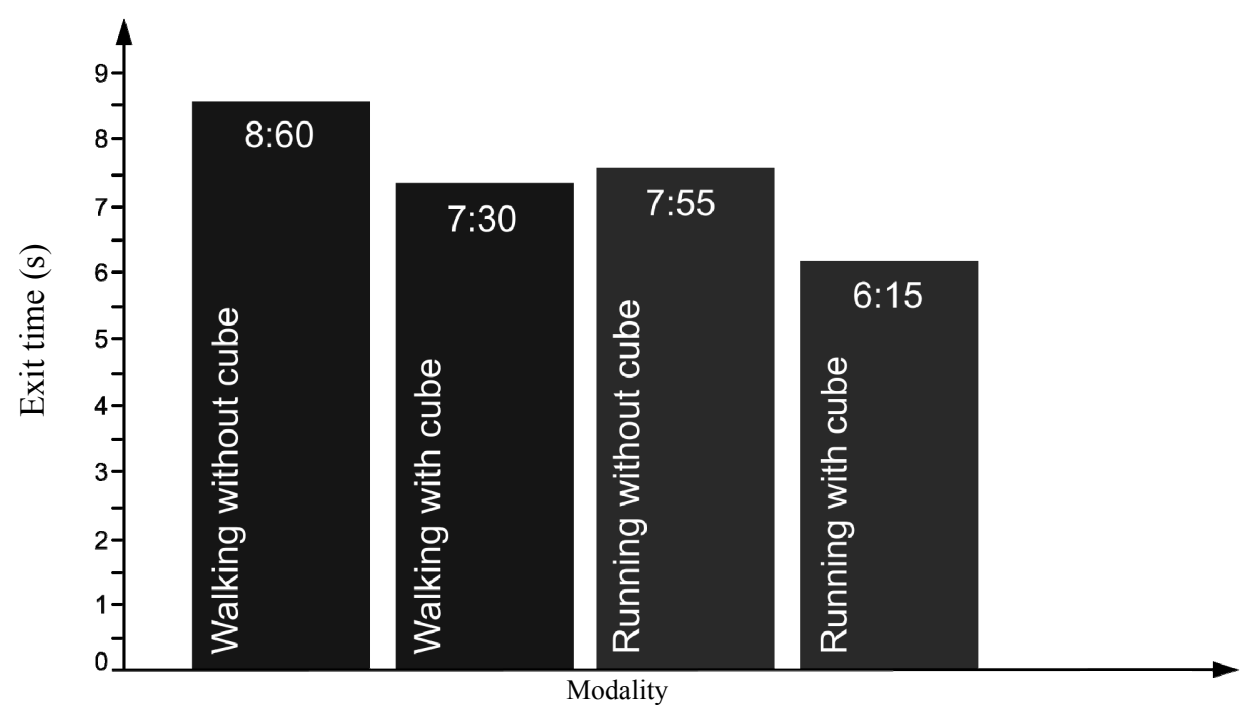

Fig. 6 Average exit time of students crowds from the outlet road (walking and running, with and without cube). Source: by M. Conti.

\section{Conclusions and Perspectives}

This learning path should be considered as an initial survey of a new intervention procedure in ecological research. The experiences of traffic psychology can be combined with the methodology of the active involvement of young people in the processes of meta-reflection.

Being brought into contact with the results of an objectively measurable experience, immersing them in the experiment, and then reflecting together on the reasons for correct behavior in traffic, is a new experience for the students. Realizing experimentally that good reasons for such behavior (moderate speed and avoidance of impacts) are derived from physical laws and not from unjustified rules imposed by the highway code, the students really understand these rules in a conscious way.

After this experience, students discover that their hypothesis was wrong. Their convictions remain unchanged: just watching the clock. Then we have discussed about whether the obstacle, experienced in a naive view as a factor of disturbance, can effectively facilitate the exit of the elements because it reduces the interactions. It was a counter-intuitive physical principle and, for this reason, not to guess in theory, but verify from the phenomenological point of view.

Participation in this educational activity allows:
- to make it clear to the students that knowledge can be built in an active way;

- to teach the students to explore the everyday contexts, reasoning about the experiences;

- to understand the adolescent which must be able to find, use and evaluate knowledge and information even if they are in conflict, which must be able to deal with, and not ignore the cognitive conflict.

The fact that an obstacle allowed to reduce total evacuation from a closed path, and that the increase in the speed of the crowd involves a greater probability of congestion-important aspects in the design of road networks and transport infrastructure-has intrigued the kids and has also motivated them to think about more general aspects of physics.

Road safety education can be considered a context for establishing a continuous link among different subjects, different school levels and for producing innovation in teaching methods. Active involvement of pupils in learning by scientific activities is proved to be very useful from the point of view of teaching. The innovative multidisciplinary approach in mobility education, introduced by project "SicuraMENTE", has proved so very effective from the didactical point of view.

Road safety education can become the context and objective of learning, while road can become the 
laboratory, so scientific research becomes the learning strategy.

\section{Acknowledgments}

Certain parts of the research in this paper were presented at 26th Workshop of ICTCT (International Co-operation on Theories and Concepts in Traffic Safety) "Sustainable and Safe Road Design from a Human Behavior Point of View - Challenges for Interdisciplinary Work in Road Safety”, in October 2013.

\section{References}

[1] Kachroo, P., Al-Nasur, S. J., Wadoo, S. A., and Shende, A. 2008. "Pedestrian Dynamics-Feedback Control of Crowd Evacuation.” In Understanding Complex Systems, edited by Abarbanel, H., Braha, D., Érdi, P., Friston, K., Haken, H., Jirsa, V., et al. UK: IOP Publishing Bristol.

[2] May, A. C. 1990. Traffic Flow Fundamental. New Jersey: Prentice Hall.

[3] Leutzbach, W. 1988. Introduction to the Theory of Traffic Flow. London: Methuen.

[4] McGuffee, S. R., and Elcock, A. H. 2010. "Diffusion, Crowding and Protein Stability in a Dynamic Molecular Model of the Bacterial Cytoplasm.” PLOS Computational Biology 6 (3). Accessed July 7, 2015. http://journals.plos.org/ploscompbiol/article?id=10.1371/j ournal.pcbi.1000694\#abstract0.

[5] Helbing, D. 1992. "A Fluid-Dynamic Model for the Movement of Pedestrians.” Complex Systems 6: 391-415.

[6] Helbing, D., and Molnar, P. 1997. "Self-Organization Phenomena in Pedestrian Crowds.” ArXiv. Accessed July 7, 2015. http://arxiv.org/pdf/cond-mat/9806152.pdf.

[7] Helbing, D. 1994. "Computer Simulation of Pedestrian Dynamics and Trail Formation.” Evolution of Natural Science 230: 229-34.

[8] Banks, J. H. 1992. "Freeway Speed-Flow-Concentration Relationships: More Evidence and Interpretations.” Transportation Research Record 1225: 53-60.

[9] Disbro, J. E., and Frame, M. 1992. "Traffic Flow Theory and Chaotic Behavior.” Transportation Research Record 1225: 109-15.

[10] Gilchrist, R. S., and Hall, F. 1992. "Three-Dimensional Relationships among Traffic Flow Theory Variables.” Transportation Research Record 1225: 99-108.

[11] Da Silva, A. J., and Stošic, B. 2010. "Critical Density of Urban Traffic.” Physics and Society. Accessed July 7, 2015. http://arxiv.org/pdf/1009.2180v1.pdf.
[12] Italian Highway Code. 2015. "Italian Highway Code. Art. 230 Law, 27/02/2015. No. 11.” Official Gazette, February 28.

[13] Duit, R., Gropengießer, H., and Kattmann, U. 2005. “Towards Science Education Research That Is Relevant for Improving Practice: The Model of Educational Reconstruction”. In Developing Standards in Research on Science Education, edited by Fischer, H. E. London: Taylor \& Francis, 1-9.

[14] Michelini, M., Mossenta, A., Stefanel, A., and Tamburini, L. 2012. "Context-Based Physics: Case Studies of Teacher Training and Materials for Science Education in Road Safety Education.” In Proceedings of the World Conference on Physics Education, 25-39.

[15] Lave, J. 1988. Cognition in Practice. Mind, Mathematics and Culture in Everyday Life. Cambridge: Cambridge University Press.

[16] McDermott, L., Shaffer, S. P., and Constantinou, C. P. 2000. "Preparing Teachers to Teach Physics and Physical Science by Inquiry.” Physics Education 35 (6): 411-6.

[17] Taasoobshirazi, G., and Carr, M. 2008. "A Review and Critique of Context-Based Physics Instruction and Assessment.” Educational Research Review 3: 155-7.

[18] Duit, R., Mikelskis-Seifert, S., Wodzinski, C. 2007. "Physics in Context-A Program for Improving Physics Instruction in Germany." Contributions from Science Education Research 3: 119-30.

[19] Michelini, M., Mossenta, A., Stefanel, A., and Tamburini, L. 2012. "Science Education in Road Safety Education as a Path toward Citizenship." In Proceedings of the International Symposium on Science and Technology for Development Education Citizenship and Social Justice, 75-86.

[20] Parchmann, I., and Luecken, M. 2010. “Context-Based Learning for Students and Teachers: Professional Development by Participating in School Innovation Projects.” Presented at International Seminar, Professional Reflections, National Science Learning Centre, York.

[21] PLON. 1986. Curriculum Materials. Zeist: NIB.

[22] Waltner, C., Wiesner, H., and Rachel, A. 2007. "Physics in Context-A Means to Encourage Student Interest in Physics.” Physics Education 42 (5): 502-7.

[23] Saunders, T. 2011. "The Physics of Crowds." Science in School 21: 23-7.

[24] Nishinari, K. 2012. "Old Pedestrians in the City." Presented at ALIAS Meeting Fostering Active Ageing from Cognitive Intervention to Smart Mobility, Milano, Italy.

[25] Shulman, L. S. 1986. "Those Who Understand: Knowledge Growth in Teaching.” Educational Researcher 15 (2): 4-14. 
[26] Helbing, D., Farkas, I., and Vicsek, T. 2000. "Simulating Dynamical Features of Escape Panic.” Nature 407: 487-90.

[27] Kirchner, A., Nishinari, K., and Schadschneider, A. 2003. "Friction Effect and Clogging in a Cellular Automaton
Model for Pedestrian Dynamics.” Physics Review E 67: 056122.

[28] Yanagisawa, D., and Nishinari, K. 2007. "Mean Field Theory for Pedestrian Outflow through an Exit.” Physical Review E 76: 061117. 